\title{
THE RING OF REAL-VALUED MULTIVARIATE POLYNOMIALS: AN ANALYST'S PERSPECTIVE
}

\author{
RAYMOND MORTINI AND RUDOLF RUPP
}

\begin{abstract}
In this survey we determine an explicit set of generators of the maximal ideals in the ring $\mathbb{R}\left[x_{1}, \ldots, x_{n}\right]$ of polynomials in $n$ variables with real coefficients and give an easy analytic proof of the Bass-Vasershtein theorem on the Bass stable rank of $\mathbb{R}\left[x_{1}, \ldots, x_{n}\right]$. The ingredients of the proof stem from different publications by Coquand, Lombardi, Estes and Ohm. We conclude with a calculation of the topological stable rank of $\mathbb{R}\left[x_{1}, \ldots, x_{n}\right]$, which seems to be unknown so far.
\end{abstract}

\section{INTRODUCTION}

In his seminal paper [14, Theorem 8], that paved the way to all future investigations of the Bass stable rank for function algebras, L. Vasershtein deduced from a Theorem of $\mathrm{H}$. Bass [2] (see below) that the Bass stable rank of the ring of real polynomials in $n$ variables is $n+1$. Since for an analyst Bass' fundamental paper is very hard to understand it is desirable to develop an analytic proof of Bass' important result that can easily be read. This was done in a paper by Estes and Ohm [4]. The whole depends on the determination of the Krull dimension of $\mathbb{R}\left[x_{1}, \ldots, x_{n}\right]$, the known proofs prior to 2005 were rather involved. But also here, a nice elementary proof had been developed around 2005 by Coquand and Lombardi [3]. Their short proof depends on the standard algebraic tool of "localization of rings". We shall replace this by a direct construction of a chain of prime ideals of length $n$ and obtain in this way an entirely analytic proof of the Bass-Vasershtein Theorem. The only tool used in the proof will now be Zorn's Lemma. In our survey we present all these proofs so that it will be entirely self-contained; it will no longer be necessary to look up half a dozen papers in order to admire this nice result by Bass and Vasershtein. We conclude the paper with a result we could not trace in the literature: the determination of the topological stable rank of $\mathbb{R}\left[x_{1}, \ldots, x_{n}\right]$ : every $(n+1)$-tuple of real-valued polynomials can be uniformly approximated on $\mathbb{R}^{n}$ be invertible $(n+1)$-tuples in $\mathbb{R}\left[x_{1}, \ldots, x_{n}\right]$.

This survey forms part of an ongoing textbook project on stable ranks of function algebras, due to be finished only in a couple of years from now (now $=2013$ ). Therefore we decided to make this chapter already available to the mathematical community (mainly for readers of this Proceedings and for master students interested in function theory and function algebras).

1991 Mathematics Subject Classification. Primary 46E25, Secondary 13M10, 26C99.

Key words and phrases. ring of real polynomials; Bass stable rank; topological stable rank; prime ideals; Krull dimension. 


\section{The maximal ideals of $\mathbb{R}\left[x_{1}, \ldots, x_{n}\right]$}

Associated with $\mathbb{R}\left[x_{1}, \ldots, x_{n}\right]$ is the following algebra of real-symmetric polynomials: $\mathbb{C}_{\text {sym }}\left[z_{1}, \ldots, z_{n}\right]=\left\{f \in \mathbb{C}\left[z_{1}, \ldots, z_{n}\right]: f\left(z_{1}, \ldots, z_{n}\right)=\overline{f\left(\bar{z}_{1}, \ldots, \bar{z}_{n}\right)} \forall\left(z_{1}, \ldots, z_{n}\right) \in \mathbb{C}^{n}\right\}$.

For shortness we write $\boldsymbol{z}$ for the $n$-tuples $\left(z_{1}, \ldots, z_{n}\right)$ and $\bar{z}$ for $\left(\bar{z}_{1}, \ldots, \bar{z}_{n}\right)$.

Lemma 1.1. $\mathbb{C}_{\mathrm{sym}}\left[z_{1}, \ldots, z_{n}\right]$ is a real algebra of complex-valued polynomials that is realisomorphic to $\mathbb{R}\left[x_{1}, \ldots, x_{n}\right]$.

Proof. It is easy to see that $\mathbb{C}_{\text {sym }}[\boldsymbol{z}]$ is a real algebra. Let $\rho: \mathbb{C}_{\text {sym }}[\boldsymbol{z}] \rightarrow \mathbb{R}[\boldsymbol{x}]$ be the restriction map $\left.p \mapsto p\right|_{\mathbb{R}^{n}}$. Note that $\rho$ is well defined, since the coefficients of a polynomial in $\mathbb{C}_{\text {sym }}[\boldsymbol{z}]$ are real; in fact if

$$
p(\boldsymbol{z})=\sum_{\boldsymbol{n} \in I} a_{\boldsymbol{n}} \mathbf{z}^{\boldsymbol{n}} \in \mathbb{C}_{\mathrm{sym}}[\boldsymbol{z}]
$$

then

$$
\overline{p(\overline{\boldsymbol{z}})}=\sum_{n \in I} \overline{a_{\boldsymbol{n}}} \mathbf{z}^{n}=\sum_{\boldsymbol{n} \in I} a_{\boldsymbol{n}} \mathbf{z}^{n} .
$$

The uniqueness of the coefficients implies that $\overline{a_{n}}=a_{n}$. Hence $a_{n} \in \mathbb{R}$. The rest is clear.

For $\boldsymbol{a} \in \mathbb{C}^{n}$ let

$$
M_{\boldsymbol{a}}:=\left\{p \in \mathbb{C}\left[z_{1}, \ldots, z_{n}\right]: p(\boldsymbol{a})=0\right\} .
$$

By Hilbert's Nullstellensatz (see for instance [9] or [8]) an ideal in $\mathbb{C}\left[z_{1}, \ldots, z_{n}\right]$ is maximal if and only if it has the form $M_{\boldsymbol{a}}$ for some $\boldsymbol{a} \in \mathbb{C}^{n}$. This will be used in the following to determine the class of maximal ideals in $\mathbb{C}_{\mathrm{sym}}\left[z_{1}, \ldots, z_{n}\right]$.

Theorem 1.2. The class of maximal ideals of $\mathbb{C}_{\mathrm{sym}}\left[z_{1}, \ldots, z_{n}\right]$ coincides with the class of ideals of the form

$$
S_{\boldsymbol{a}}:=M_{\boldsymbol{a}} \cap M_{\overline{\boldsymbol{a}}} \cap \mathbb{C}_{\mathrm{sym}}\left[z_{1}, \ldots, z_{n}\right],
$$

where $\boldsymbol{a} \in \mathbb{C}^{n}$. The set $\{\boldsymbol{a}, \overline{\boldsymbol{a}}\}$ is uniquely determined for a given maximal ideal.

Proof. We first note that $M_{\boldsymbol{a}} \cap M_{\overline{\boldsymbol{a}}} \cap \mathbb{C}_{\text {sym }}[\boldsymbol{z}]=M_{\boldsymbol{a}} \cap \mathbb{C}_{\text {sym }}[\boldsymbol{z}]$, because for every polynomial $p$ in $\mathbb{C}_{\text {sym }}[\boldsymbol{z}]$ it holds that $p(\boldsymbol{a})=0$ if and only if $p(\overline{\boldsymbol{a}})=0$.

Next we show that the ideals $S_{\boldsymbol{a}}$ are maximal. So suppose that $f \in \mathbb{C}_{\mathrm{sym}}[\boldsymbol{z}]$ does not vanish at $\boldsymbol{a}$. Then

$$
(f-f(\boldsymbol{a}))(f-\overline{f(\boldsymbol{a})})=f^{2}-(2 \operatorname{Re} f(\boldsymbol{a})) f+|f(\boldsymbol{a})|^{2} \in S_{\boldsymbol{a}}
$$

and

$$
1=\frac{(f-f(\boldsymbol{a}))(f-\overline{f(\boldsymbol{a})})}{|f(\boldsymbol{a})|^{2}}-f \frac{f-(f(\boldsymbol{a})+\overline{f(\boldsymbol{a})})}{|f(\boldsymbol{a})|^{2}} .
$$

Hence the ideal, $I_{\mathbb{C}_{\mathrm{sym}}[\boldsymbol{z}]}\left(S_{\boldsymbol{a}}, f\right)$, generated by $S_{\boldsymbol{a}}$ and $f$ is the whole algebra and so $S_{\boldsymbol{a}}$ is maximal. We note that in the case where $f(\boldsymbol{a})$ is real, we simply could argue as follows, since the constant functions $\boldsymbol{z} \mapsto f(\boldsymbol{a})$ and $\boldsymbol{z} \mapsto 1 / f(\boldsymbol{a})$ then belong to $\mathbb{C}_{\text {sym }}[\boldsymbol{z}]$ :

$$
1=-\frac{f-f(\boldsymbol{a})}{f(\boldsymbol{a})}+\frac{f}{f(\boldsymbol{a})} \in I_{\mathbb{C}_{\mathrm{sym}}[\boldsymbol{z}]}\left(S_{\boldsymbol{a}}, f\right) .
$$


It remains to show that every maximal ideal $M$ in $\mathbb{C}_{\text {sym }}[\boldsymbol{z}]$ coincides with $S_{\boldsymbol{a}}$ for some $\boldsymbol{a} \in \mathbb{C}^{n}$. Suppose, to the contrary, that $M$ is not contained in any ideal of the form $S_{\boldsymbol{a}}$. Hence, for every $\boldsymbol{a} \in \mathbb{C}^{n}$, there is $p_{\boldsymbol{a}} \in M$ such that $p_{\boldsymbol{a}}(\boldsymbol{a}) \neq 0$. By Hilbert's Nullstellensatz, the ideal generated by the set $S=\left\{p_{\boldsymbol{a}}: \boldsymbol{a} \in \mathbb{C}^{n}\right\}$ in $\mathbb{C}[\boldsymbol{z}]$ coincides with $\mathbb{C}[\boldsymbol{z}]$. Hence there are $q_{j} \in \mathbb{C}[\boldsymbol{z}]$ and finitely many $\boldsymbol{a}_{j} \in \mathbb{C}^{n},(j=1, \ldots, N)$, such that

$$
\sum_{j=1}^{N} q_{j} p_{\boldsymbol{a}_{j}}=1 .
$$

Now, by taking complex conjugates, and using the fact that $p_{\boldsymbol{a}_{j}} \in M \subseteq \mathbb{C}_{\mathrm{sym}}[\boldsymbol{z}]$, we get

$$
1=\overline{\sum_{j=1}^{N} q_{j}(\overline{\boldsymbol{z}}) p_{\boldsymbol{a}_{j}}(\overline{\boldsymbol{z}})}=\sum_{j=1} \overline{q_{j}(\overline{\boldsymbol{z}})} p_{\boldsymbol{a}_{j}}(\boldsymbol{z}) .
$$

Hence, with

$$
q_{j}^{*}(\boldsymbol{z})=\frac{1}{2}\left(\overline{q_{j}(\overline{\boldsymbol{z}})}+q_{j}(\boldsymbol{z})\right)
$$

we conclude that

$$
\sum_{j=1}^{N} q_{j}^{*} p_{\boldsymbol{a}_{j}}=1 .
$$

Since $q_{j}^{*} \in \mathbb{C}_{\text {sym }}[\boldsymbol{z}]$ and $p_{\boldsymbol{a}_{j}} \in M$ we obtain the contradiction that $1 \in M$. Thus $M \subseteq S_{\boldsymbol{a}}$ for some $\boldsymbol{a} \in \mathbb{C}^{n}$. The maximality of $M$ now implies that $M=S_{\boldsymbol{a}}$.

Finally we show the uniqueness of $\{\boldsymbol{a}, \overline{\boldsymbol{a}}\}$. So suppose that $\boldsymbol{b} \notin\{\boldsymbol{a}, \overline{\boldsymbol{a}}\}$.

Case 1 There is an index $i_{0}$ such that $b_{i_{0}} \notin\left\{a_{i_{0}}, \overline{a_{i_{0}}}\right\}$. Then the polynomial $p$, given by

$$
p\left(z_{1}, \ldots, z_{n}\right)=\left(z_{i_{0}}-a_{i_{0}}\right)\left(z_{i_{0}}-\overline{a_{i_{0}}}\right)
$$

belongs to $\mathbb{C}_{\mathrm{sym}}\left[z_{1}, \ldots, z_{n}\right]$, vanishes at $\boldsymbol{a}$, but not at $\boldsymbol{b}$ (if $a_{i_{0}} \in \mathbb{R}$, then it suffices to take $\left.p\left(z_{1}, \ldots, z_{n}\right)=z_{i_{0}}-a_{i_{0}}\right)$.

Case 2 There are two indices $i_{0}$ and $i_{1}$ such that $a_{i_{\nu}} \notin \mathbb{R}, b_{i_{0}}=\overline{a_{i_{0}}}$ and $b_{i_{1}}=a_{i_{1}}$. Then the polynomial $q$ given by

$$
q\left(z_{1}, \ldots, z_{n}\right)=\left(\left(z_{i_{0}}-a_{i_{0}}\right)+\left(z_{i_{1}}-a_{i_{1}}\right)\right) \cdot\left(\left(z_{i_{0}}-\overline{a_{i_{0}}}\right)+\left(z_{i_{1}}-\overline{a_{i_{1}}}\right)\right)
$$

belongs to $\mathbb{C}_{\mathrm{sym}}\left[z_{1}, \ldots, z_{n}\right]$, vanishes at $\boldsymbol{a}$, but not at $\boldsymbol{b}$.

Hence, in both cases, $p \in S_{\boldsymbol{a}} \backslash S_{\boldsymbol{b}}$. There are no other cases left.

Theorem 1.3. The Bézout equation $\sum_{j=1}^{k} q_{j} p_{j}=1$ admits a solution in the ring $R=$ $\mathbb{C}_{\mathrm{sym}}\left[z_{1}, \ldots, z_{n}\right]$ or $\mathbb{R}\left[x_{1}, \ldots, x_{n}\right]$ if and only if the polynomials $p_{j}$ do not have a common zero in $\mathbb{C}^{n}$.

Proof. By the identity theorem for holomorphic functions of several complex variables, the condition $\sum_{j=1}^{k} q_{j} p_{j}=1$ on $\mathbb{R}^{n}$ implies that the same equality holds on $\mathbb{C}^{n}$. Hence the given polynomials $p_{j}$ do not have a common zero in $\mathbb{C}^{n}$.

Conversely, if the $p_{j}$ do not have a common zero in $\mathbb{C}^{n}$ then, by Theorem 1.2 , the ideal generated by the $p_{j}$ in $R$ cannot be a proper ideal. Hence there are $q_{j} \in R$ such that $\sum_{j=1}^{k} q_{j} p_{j}=1$. 
We shall now determine an explicit class of generators for the maximal ideals in $\mathbb{R}\left[x_{1}, \ldots, x_{n}\right]$. Recall that by Hilbert's Nullstellensatz the maximal ideals in $\mathbb{C}\left[z_{1}, \ldots, z_{n}\right]$ are generated by $n$ polynomials of the form $z_{1}-a_{1}, \ldots, z_{n}-a_{n}$, where $\boldsymbol{a}:=\left(a_{1}, \ldots, a_{n}\right) \in \mathbb{C}^{n}$. The situation for the real algebra $\mathbb{R}\left[x_{1}, \ldots, x_{n}\right]$ is quite different. Here are some examples that will reflect the general situation dealt with below. We identify $\mathbb{R}\left[x_{1}, \ldots, x_{n}\right]$ with $\mathbb{C}_{\text {sym }}\left[z_{1}, \ldots, z_{n}\right]$. The proof of the assertions is left as an exercise to the reader.

\section{Example 1.4.}

(1) Let $\sigma \in \mathbb{C} \backslash \mathbb{R}$ and $r_{j} \in \mathbb{R}, j=1,2, \ldots, n-1$. Then the ideal generated by

$$
x_{n}^{2}-(2 \operatorname{Re} \sigma) x_{n}+|\sigma|^{2}
$$

and $x_{j}-r_{j},(j=1, \ldots, n-1)$, is maximal in $\mathbb{R}\left[x_{1}, \ldots, x_{n}\right]$. It corresponds to the ideal $S_{\left(r_{1}, \ldots, r_{n-1}, \sigma\right)}$.

(2) The ideal $I_{\mathbb{R}[x, y]}\left(1+x^{2}, 1+y^{2}\right)$ generated by $1+x^{2}$ and $1+y^{2}$ is not maximal.

(4) The ideal $M:=I_{\mathbb{R}[x, y]}\left(1+x^{2}, 1+y^{2}, 1+x y, x-y\right)$ is maximal and corresponds to $S_{(i, i)}$.

(5) The following representations hold:

$$
\begin{aligned}
M & =I_{\mathbb{R}[x, y]}\left(1+x^{2}, 1+y^{2}, x-y\right) \\
& =I_{\mathbb{R}[x, y]}\left(1+x^{2}, 1+y^{2}, 1+x y\right) \\
& =I_{\mathbb{R}[x, y]}(1+x y, x-y) .
\end{aligned}
$$

Theorem 1.5. Modulo a re-enumeration of the indices, the maximal ideals $M$ of $R:=$ $\mathbb{R}\left[x_{1}, \ldots, x_{n}\right]$ are generated by polynomials of the form

$$
\begin{gathered}
p_{j}:=x_{j}-r_{j},(j=1, \ldots, k), \\
p_{k+j}:=x_{k+j}^{2}-\left(2 \operatorname{Re} a_{j}\right) x_{k+j}+\left|a_{j}\right|^{2},(j=1, \ldots, m)
\end{gathered}
$$

$\left(r_{j} \in \mathbb{R}, a_{j} \in \mathbb{C} \backslash \mathbb{R}, k+m=n\right)$, and $2^{n-k}-2$ multilinear polynomials $q_{j}$ in $\mathbb{R}\left[x_{k+1}, \ldots, x_{n}\right]$ vanshing at $a_{k+1}, \ldots, a_{n}$. More precisely, we have

$$
M=\sum_{j=1}^{n} p_{j}\left(x_{j}\right) R+\sum_{j=1}^{2^{n-k}-2} q_{j}\left(x_{k+1}, \ldots, x_{n}\right) \mathbb{R} .
$$

Proof. Since $\mathbb{R}\left[x_{1}, \ldots, x_{n}\right]$ is isomorphic to $S:=\mathbb{C}_{\mathrm{sym}}\left[z_{1}, \ldots, z_{n}\right]$, it suffices to show that every maximal ideal $S_{\boldsymbol{a}}$ in $S$ is generated by polynomials of the desired type (Theorem 1.2). Fix $\boldsymbol{a} \in \mathbb{C}^{n}$. We may assume that

$$
\boldsymbol{a}=\left(r_{1}, \ldots, r_{k}, a_{k+1}, \ldots, a_{k+m}\right),
$$

with $k+m=n$, where $r_{j} \in \mathbb{R}$ and $a_{k+1}, \ldots, a_{k+m} \in \mathbb{C} \backslash \mathbb{R}$. Note that $k$ or $m$ may be 0 . Let $f \in S_{\boldsymbol{a}}$ and $\boldsymbol{z}=\left(z_{1}, \ldots, z_{n}\right)$. By the Euclidean division procedure

$$
f(\boldsymbol{z})=\sum_{j=1}^{n} p_{j}(\boldsymbol{z}) q_{j}(\boldsymbol{z})+r\left(z_{k+1}, \ldots, z_{k+m}\right),
$$


where $\operatorname{deg}_{z_{j}} r<2$ for $k+1 \leq j \leq k+m=n$. Hence $r$ is a multilinear polynomial of the form

$$
r\left(z_{k+1}, \ldots, z_{k+m}\right)=\sum_{\boldsymbol{j}} c_{\boldsymbol{j}} z_{k+1}^{j_{1}} \cdots z_{k+m}^{j_{m}}
$$

$\boldsymbol{j}=\left(j_{1}, \ldots, j_{m}\right), j_{\ell} \in\{0,1\}, c_{\boldsymbol{j}} \in \mathbb{R}$. Moreover, $r\left(a_{k+1}, \ldots, a_{k+m}\right)=f(\boldsymbol{a})=0$. Now the real vector-space, $V$, of all multilinear real-symmetric polynomials in $m$ variables has the algebraic dimension $2^{m}$. Hence, the subspace $V^{*}$ of all $p \in V$ with $p\left(a_{k+1}, \ldots, a_{k+m}\right)=$ $p\left(\overline{a_{k+1}}, \ldots, \overline{a_{k+m}}\right)=0$ has dimension $2^{m}-2$. Let $\left\{q_{1}, \ldots, q_{2^{m}-2}\right\}$ be a basis of $V^{*}$. Then

$$
f \in \sum_{j=1}^{n}\left(p_{j} R\right)+\sum_{j=1}^{2^{m}-2}\left(q_{j} \mathbb{R}\right) .
$$

We shall now unveil an explicit basis for $V^{*}$ whenever $a_{j}=i$ for every $j$.

Lemma 1.6. Let $\boldsymbol{i}=(i, \ldots, i) \in \mathbb{C}^{m}$. Then a (vector-space) basis of

$$
V^{*}=\left\{f\left(z_{1}, \ldots, z_{m}\right)=\sum_{j_{1}, \ldots, j_{m}} c_{\boldsymbol{j}} z_{k+1}^{j_{1}} \cdots z_{k+m}^{j_{m}}, j_{\ell} \in\{0,1\}, c_{\boldsymbol{j}} \in \mathbb{R}, f(\boldsymbol{i})=0\right\}
$$

is given by

$$
\begin{array}{rl}
x_{1}-x_{j} & 1<j \leq m \\
1+x_{j_{1}} x_{j_{2}} & 1 \leq j_{1}<j_{2} \leq m \\
x_{1}+x_{j_{1}} x_{j_{2}} x_{j_{3}} & 1 \leq j_{1}<j_{2}<j_{3} \leq m \\
1-\prod_{\ell=1}^{4} x_{j_{\ell}} & 1 \leq j_{1}<\cdots<j_{4} \leq m \\
x_{1}-\prod_{\ell=1}^{5} x_{j_{\ell}} & 1 \leq j_{1}<\cdots<j_{5} \leq m \\
\ldots \ldots & \ldots \ldots .
\end{array}
$$

The last element has exactly one of the following forms:

$$
\left\{\begin{array}{lll}
x_{1}-\prod_{j=1}^{m} x_{j} & \text { if } m \equiv 1 & \bmod 4 \\
1+\prod_{j=1}^{m} x_{j} & \text { if } m \equiv 2 & \bmod 4 \\
x_{1}+\prod_{j=1}^{m} x_{j} & \text { if } m \equiv 3 & \bmod 4 \\
1-\prod_{j=1}^{m} x_{j} & \text { if } m \equiv 0 & \bmod 4
\end{array}\right.
$$

Proof. All the polynomials above vanish at $\boldsymbol{i}$ (this is the reason for their cyclic behaviour mod 4). Moreover, they are linear independent and there are exactly $2^{m}-2$ of them. Note that the second summand has the form

$$
x_{1}^{\varepsilon_{1}} x_{2}^{\varepsilon_{2}} \cdots x_{m}^{\varepsilon_{m}}, \quad \varepsilon_{j} \in\{0,1\},
$$

the monomials $1=\prod_{j=1}^{m} x_{j}^{0}$ and $x_{1}=x_{1} \prod_{j=2}^{m} x_{j}^{0}$ being excluded. 
We conclude this section with the final form of the generators of the maximal ideals in $\mathbb{R}\left[x_{1}, \ldots, x_{n}\right]$.

Theorem 1.7. Let $m+k=n, m \geq 2$, and $\boldsymbol{a}:=\left(i, \ldots, i, r_{m+1}, \ldots, r_{m+k}\right) \in \mathbb{C}^{m} \times \mathbb{R}^{k}$. The maximal ideal $S_{\boldsymbol{a}}$ of $\mathbb{R}\left[x_{1}, \ldots, x_{n}\right]$ is generated by the $2^{m}-2$ multilinear polynomials in Lemma 1.6 and the polynomials

$$
p_{m+j}:=x_{m+j}-r_{m+j},(j=1, \ldots, k) .
$$

Proof. Using Theorem 1.5, it suffices to show that the quadratic polynomials $1+x_{j}^{2}$, $j=1, \ldots, m$, belong to the ideal generated by the $2^{m}-2$ multilinear polynomials in Lemma 1.6. This is clear, however, in view of the following relations:

$$
\begin{gathered}
1+x_{j}^{2}=-\left(x_{1}-x_{j}\right) x_{j}+\left(1+x_{1} x_{j}\right) \text { for } j=2, \ldots, m, \\
1+x_{1}^{2}=\left(x_{1}-x_{m}\right) x_{1}+\left(1+x_{1} x_{m}\right) .
\end{gathered}
$$

The general case of an arbitrary maximal ideal $S_{\boldsymbol{a}}$ is easily deduced by using the transformation

of $\mathbb{C}^{m}$ onto $\mathbb{C}^{m}$, whenever

$$
\chi\left(z_{1}, \ldots, z_{m}\right)=\left(\frac{z_{1}-\alpha_{1}}{\beta_{1}}, \ldots, \frac{z_{m}-\alpha_{m}}{\beta_{m}}\right)
$$

$$
\boldsymbol{a}=\left(\alpha_{1}+i \beta_{1}, \ldots, \alpha_{m}+i \beta_{m}, r_{m+1}, \ldots, r_{m+k}\right) \in \mathbb{C}^{m} \times \mathbb{R}^{k} \subseteq \mathbb{C}^{n},
$$

with $\beta_{j} \neq 0$ for $j=1, \ldots, m$.

Using more algebraic methods, it can be shown, that every maximal ideal in $R:=$ $F\left[x_{1}, \ldots, x_{n}\right]$ is generated by $n$ elements (see [8, p. 20]), where $F$ is a field. Finally, let us mention that $R$ is a Noetherian ring (this means that every ideal in $R$ is finitely generated; this is Hilbert's basis theorem).

\section{The Bass stable Rank of $\mathbb{R}\left[x_{1}, \ldots, x_{n}\right]$}

Definition 2.1. Let $R$ be a commutative unital ring with identity element 1 . We assume that $1 \neq 0$, that is $R$ is not the trivial ring $\{0\}$.

(1) If $a_{j} \in R,(j=1, \ldots, n)$, then

$$
I_{R}\left(a_{1}, \ldots, a_{n}\right):=\left\{\sum_{j=1}^{n} x_{j} a_{j}: x_{j} \in R\right\}
$$

is the ideal generated by the $a_{j}$ in $R$.

(2) An $n$-tuple $\left(f_{1}, \ldots, f_{n}\right) \in R^{n}$ is said to be invertible (or unimodular), if there exists $\left(x_{1}, \ldots, x_{n}\right) \in R^{n}$ such that the Bézout equation $\sum_{j=1}^{n} x_{j} f_{j}=1$ is satisfied. The set of all invertible $n$-tuples is denoted by $U_{n}(R)$. Note that $U_{1}(R)=R^{-1}$. An $(n+1)$-tuple $\left(f_{1}, \ldots, f_{n}, g\right) \in U_{n+1}(R)$ is called reducible if there exists $\left(a_{1}, \ldots, a_{n}\right) \in$ $R^{n}$ such that $\left(f_{1}+a_{1} g, \ldots, f_{n}+a_{n} g\right) \in U_{n}(R)$.

(3) The Bass stable rank of $R$, denoted by bsr $R$, is the smallest integer $n$ such that every element in $U_{n+1}(R)$ is reducible. If no such $n$ exists, then bsr $R=\infty$. 
Note that if bsr $R=n, n<\infty$, and $m \geq n$, then every invertible $(m+1)$-tuple $(\boldsymbol{f}, g) \in R^{m+1}$ is reducible [14, Theorem 1].

Let $\mathbb{N}=\{0,1,2, \ldots\}$. For the sequel, we make the convention that the symbol $\subset$ denotes strict inclusion.

Definition 2.2. Let $R$ be a commutative unital ring, $R \neq\{0\}$.

(1) A chain $\mathfrak{C}=\left\{I_{0}, I_{1}, \ldots, I_{n}\right\}$ of ideals in $R$ is said to have length $n(n \in \mathbb{N})$, if

$$
I_{0} \subset I_{1} \subset \cdots \subset I_{n}
$$

the inclusions being strict. We also call $\mathfrak{C}$ an $n$-chain. Note the length of a chain $\mathfrak{C}$ counts the number of strict inclusions between the members of $\mathfrak{C}$ and not the cardinal of $\mathfrak{C}$.

(2) The Krull dimension, $\operatorname{Krd} R$, of $R$ is defined to be the supremum of the lengths of all increasing chains

$$
P_{0} \subset \cdots \subset P_{n}
$$

of prime ideals in $R$. Note that $\operatorname{Krd} R \in\{0,1, \ldots, \infty\}$.

Here is now Vasershtein's theorem (and its proof sketched in [14]).

Theorem 2.3 (Vasershtein).

$$
\operatorname{bsr} \mathbb{R}\left[x_{1}, \ldots, x_{n}\right]=n+1 .
$$

Proof. We first show that $\operatorname{bsr} \mathbb{R}\left[x_{1}, \ldots, x_{n}\right] \geq n+1$. Consider the invertible $(n+1)$-tuple

$$
\left(x_{1}, \ldots, x_{n}, 1-\sum_{j=1}^{n} x_{j}^{2}\right)
$$

in $\mathbb{R}\left[x_{1}, \ldots, x_{n}\right]$. This tuple cannot be reducible in $\mathbb{R}\left[x_{1}, \ldots, x_{n}\right] \subseteq C\left(\mathbb{R}^{n}, \mathbb{R}\right)$, since otherwise the $n$-tuple $\left(x_{1}, \ldots, x_{n}\right)$, restricted to the unit sphere $\partial \mathbf{B}$ in $\mathbb{R}^{n}$, would have a zero-free extension $\boldsymbol{e}$ to the unit ball $\mathbf{B}$, where $\boldsymbol{e}$ is given by

$$
\left(x_{1}+u_{1} \cdot\left(1-\sum_{j=1}^{n} x_{j}^{2}\right), \ldots, x_{n}+u_{n} \cdot\left(1-\sum_{j=1}^{n} x_{j}^{2}\right)\right)
$$

for some $u_{j} \in \mathbb{R}\left[x_{1}, \ldots, x_{n}\right]$. This means that $\boldsymbol{e}$ does not take the value $(0, \ldots, 0)$ on $\mathbf{B}$. This contradicts Brouwer's result that the identity map

$$
\left(x_{1}, \ldots, x_{n}\right): \partial \mathbf{B} \rightarrow \partial \mathbf{B}
$$

defined on the boundary of the closed unit ball $\mathbf{B}$ in $\mathbb{R}^{n}$ does not admit a zero-free continuous extension to $\mathbf{B}$.

Next we prove that $\operatorname{bsr} \mathbb{R}\left[x_{1}, \ldots, x_{n}\right] \leq n+1$. This follows from a combination of Theorem 5.6 below, telling us that the Bass stable rank of a Noetherian ring with Krull dimension $n$ is less than or equal to $n+1$, and Theorem 3.9, according to which the Krull dimension of $\mathbb{R}\left[x_{1}, \ldots, x_{n}\right]$ is $n$.

In the next section we shall now present analytic proofs of both Theorems mentioned above. They were given by Estes and Ohm for Theorem 5.6 ([4]) and Coquand and Lombardi for Theorem 3.9 ([3]). 
Remark 2.4. Concerning the polynomial ring $\mathbb{C}\left[z_{1}, \ldots, z_{n}\right]$, to the best of our knowledge, the exact value of the Bass stable rank for $\mathbb{C}\left[z_{1}, \ldots, z_{n}\right]$ is not yet known. Only estimates are available:

$$
\operatorname{bsr} \mathbb{C}\left[z_{1}, \ldots, z_{n}\right] \leq n+1,
$$

(follows as in the proof for $\mathbb{R}\left[x_{1}, \ldots, x_{n}\right]$ because the Krull dimension of $\mathbb{C}\left[z_{1}, \ldots, z_{n}\right]$ is also $n$ by Theorem 3.9) and

$$
\operatorname{bsr} \mathbb{C}\left[z_{1}, \ldots, z_{n}\right] \geq\left\lfloor\frac{n}{2}\right\rfloor+1
$$

(see [5], [6]).

\section{The Krull dimension of $\mathbb{R}\left[x_{1}, \ldots, x_{n}\right]$}

We begin with our own proof of the Coquand-Lombardi result concerning an elementary characterization of the Krull dimension of a commutative unital ring. We avoid the algebraic tool of considering localized rings and explicitely construct (with the help of Zorn's Lemma) chains of prime ideals having the correct length. Our tool will be the following standard result, which we would like to present, too.

Lemma 3.1 (Krull). Let $R$ be a commutative unital ring, $R \neq\{0\}$, and $S$ a multiplicatively closed set in $R$ with $1 \in S$. Suppose that $I$ is an ideal in $R$ with $I \cap S=\emptyset$. Then there exists a prime ideal $P$ with $I \subseteq P$ such that $P \cap S=\emptyset$.

Proof. Let $\mathscr{V}$ be the set of all ideals $J$ with $I \subseteq J$ and $J \cap S=\emptyset$. Then $\mathscr{V} \neq \emptyset$ because $I \in \mathscr{V}$, and $\mathscr{V}$ is partially ordered by set inclusion. If $\mathfrak{C}$ is any increasing chain in $\mathscr{V}$ then $\bigcup_{J \in \mathfrak{C}} J$ obviously is an ideal belonging to $\mathscr{V}$. Hence, by Zorn's Lemma, $\mathscr{V}$ admits a maximal element $P$. Since $1 \in S$ and $S \cap P=\emptyset$, we obtain that $P \subset R$, the inclusion being strict. Moreover, $I \subseteq P$. We claim that $P$ is a prime ideal. For $f, g \in R$, let $f g \in P$ and suppose that neither $f$ nor $g$ belongs to $P$. Since $P$ is maximal in $\mathscr{V}$, the ideals $P+f R$ and $P+g R$ meet $S$. Hence there exists $s, s^{\prime} \in S, p, p^{\prime} \in P$, and $r, r^{\prime} \in R$ such that

$$
s=p+r f \text { and } s^{\prime}=p^{\prime}+r^{\prime} g .
$$

Multiplying both terms, we obtain

$$
s s^{\prime}=p p^{\prime}+\left(r^{\prime} g\right) p+(r f) p^{\prime}+r r^{\prime}(f g) \in P .
$$

Since $S$ is multiplicatively closed, $s s^{\prime} \in S$. Hence $P \cap S \neq \emptyset$; a contradiction. We conclude that $P$ is prime.

For $a, x \in R$ and $n \in \mathbb{N}$, let $L_{a, n, x}(y)=a^{n}(y+a x)$. If $a_{j}, x_{j} \in R$ and $n_{j} \in \mathbb{N}$ are given, then we abbreviate $L_{j}(y):=L_{a_{j}, n_{j}, x_{j}}(y)$.

Lemma 3.2. Let $Q_{0}$ and $Q_{1}$ be ideals in a commutative unital ring $R$ with $Q_{0} \subset Q_{1}$ and let $a \in Q_{1} \backslash Q_{0}$. Suppose that $Q_{0}$ is prime and that for $r, x \in R$ and $n \in \mathbb{N}$ we have

$$
a^{n}(r+a x) \in Q_{0} .
$$

Then $r \in Q_{1}$.

Proof. Because $a \notin Q_{0}$, the primeness of $Q_{0}$ implies that $r+a x \in Q_{0} \subseteq Q_{1}$. Since $a \in Q_{1}$, we conclude that $r \in Q_{1}$. 
Theorem 3.3 (Coquand-Lombardi). Let $R$ be a commutative unital ring, $R \neq\{0\}$. For $N \in \mathbb{N}$, the following assertions are equivalent:

(1) The Krull dimension of $R$ is at most $N$.

(2) For all $\left(a_{0}, \ldots, a_{N}\right) \in R^{N+1}$ there exists $\left(x_{0}, \ldots, x_{N}\right) \in R^{N+1}$ and $\left(n_{0}, \ldots, n_{N}\right) \in$ $\mathbb{N}^{N+1}$ such that

$$
a_{0}^{n_{0}}\left(a_{1}^{n_{1}}\left(\cdots\left(a_{N}^{n_{N}}\left(1+a_{N} x_{N}\right)+\cdots\right)\right)+a_{0} x_{0}\right)=0,
$$

in other words

Proof. We show the contraposition of the assertion.

$\neg(1) \Longrightarrow \neg(2)$ Assume that the Krull dimension of $R$ is at least $N+1$. Then $R$ admits a strictly increasing $(N+1)$-chain

$$
P_{0} \subset \cdots \subset P_{N} \subset P_{N+1}
$$

of prime ideals $P_{j}$. Choose $a_{j} \in P_{j+1} \backslash P_{j}, j=0, \ldots, N$. If we suppose, contrariwise, that (3.2) holds, then

$$
a_{0}^{n_{0}}\left(L_{1} \circ \cdots \circ L_{N}(1)+a_{0} x_{0}\right)=0 \in P_{0} .
$$

Hence, by Lemma $3.2, L_{1} \circ \cdots \circ L_{N}(1) \in P_{1}$. Now

$$
L_{1} \circ \cdots \circ L_{N}(1)=a_{1}^{n_{1}}\left(L_{2} \circ \cdots \circ L_{N}(1)+a_{1} x_{1}\right) \in P_{1} .
$$

Hence, by Lemma 3.2, $L_{2} \circ \cdots \circ L_{N}(1) \in P_{2}$. Continuing in this way, we deduce that

$$
a_{N}^{n_{N}}\left(1+a_{N} x_{N}\right)=L_{N}(1) \in P_{N} .
$$

Hence, by Lemma 3.2, $1 \in P_{N+1}$; a contradiction.

$\neg(2) \Longrightarrow \neg(1)$ Suppose that there exists $\boldsymbol{a}=\left(a_{0}, \ldots, a_{N}\right) \in R^{N+1}$ such that for all $\boldsymbol{x} \in R^{N+1}$ and $\boldsymbol{n} \in \mathbb{N}^{N+1}$ one has

$$
L_{0} \circ \cdots \circ L_{N}(1) \neq 0 .
$$

For $j=0, \ldots, N$, let

$$
S_{j}=\left\{L_{j} \circ \cdots \circ L_{N}(1): \boldsymbol{x} \in R^{N-j+1}, \boldsymbol{n} \in \mathbb{N}^{N-j+1}\right\} .
$$

Then $S_{N} \subseteq S_{N-1} \subseteq \ldots \subseteq S_{0}$, or, what is the same, $S_{0}^{c} \subseteq S_{1}^{c} \subseteq \ldots \subseteq S_{N}^{c}$ where $S^{c}$ denotes the the complement of $S$. Note that $\left\{1, a_{j}, \ldots, a_{N}\right\} \subseteq S_{j}$. We claim that $S_{j}$ is multiplicatively closed. This follows by an inductive argument on $N-j$. If $j=0$, then

$$
S_{N}=\left\{a_{N}^{n_{N}}\left(1+a_{N} x_{N}\right): x_{N} \in R, n_{N} \in \mathbb{N}\right\}
$$

is easily seen to be multiplicatively closed. If for some $j, S_{N-j}$ is multiplicatively closed, then we use that

$$
L_{N-(j+1)} \circ L_{N-j} \circ \cdots \circ L_{N}(1)=a_{N-(j+1)}^{n_{N-(j+1)}}\left(L_{N-j} \circ \cdots \circ L_{N}(1)+a_{N-(j+1)} x_{N-(j+1)}\right)
$$


and observe that

$$
a^{n}(s+a x) \cdot a^{m}\left(s^{\prime}+a x^{\prime}\right)=a^{n+m}\left(s s^{\prime}+a x^{\prime \prime}\right)
$$

where $x^{\prime \prime}=s x^{\prime}+x s^{\prime}+a x x^{\prime}$.

Step1 Looking at the zero ideal $I:=\{0\}$, and noticing that by assumption $0 \notin S_{0}$, Krull's Lemma 3.1 tells us that there exists a prime ideal $P_{0}$ with $P_{0} \cap S_{0}=\emptyset$, or in other words, $P_{0} \subseteq S_{0}^{c}$. Now $a_{0} \in S_{0}$ implies that $a_{0} \notin P_{0}$. We claim that

$$
P_{0} \subset I_{R}\left(P_{0}, a_{0}\right) \subseteq S_{1}^{c} \text {. }
$$

In fact, if the second inclusion does not hold, then there is $p_{0} \in P_{0}$ and $x \in R$ such that

$$
p_{0}+x a_{0}=L_{1} \circ \cdots \circ L_{N}(1) \in S_{1} \subseteq S_{0} .
$$

Hence

$$
\begin{aligned}
p_{0} & =L_{1} \circ \cdots \circ L_{N}(1)-x a_{0} \\
& =a_{0}^{0}\left(L_{1} \circ \cdots \circ L_{N}(1)-x a_{0}\right) \in S_{0} .
\end{aligned}
$$

Thus $p_{0} \in P_{0} \cap S_{0}$; a contradiction to the choice of $P_{0}$. Hence the inclusions (3.3) hold.

Step 2 Now we apply Krull's Lemma again to get a prime ideal $P_{1}$ with

$$
P_{0} \subset I_{R}\left(P_{0}, a_{0}\right) \subseteq P_{1} \subseteq S_{1}^{c}
$$

Observe that $a_{1} \notin P_{1}$ because $a_{1} \in S_{1}$. We claim that

$$
P_{1} \subset I_{R}\left(P_{1}, a_{1}\right) \subseteq S_{2}^{c} .
$$

In fact, if the second inclusion does not hold, then there is $p_{1} \in P_{1}$ and $x \in R$ such that

$$
p_{1}+x a_{1}=L_{2} \circ \cdots \circ L_{N}(1) \in S_{2} \subseteq S_{1} .
$$

Hence

$$
\begin{aligned}
p_{1} & =L_{2} \circ \cdots \circ L_{N}(1)-x a_{1} \\
& =a_{1}^{0}\left(L_{2} \circ \cdots \circ L_{N}(1)-x a_{1}\right) \in S_{1} .
\end{aligned}
$$

Thus $p_{1} \in P_{1} \cap S_{1}$; a contradiction to the choice of $P_{1}$. Hence the inclusions (3.4) hold.

Step $\mathbf{N}$ Continuing in this way, we get a chain of prime ideals $P_{j}, j=0, \ldots, N$, with

$$
P_{0} \subset P_{1} \subset \cdots \subset P_{N} \subseteq S_{N}^{c} .
$$

Observe that $a_{N} \notin P_{N}$ because $a_{N} \in S_{N}$. Hence $P_{N}$ is a proper ideal. Therefore, $P_{N}$ is contained in a maximal ideal $M$. We claim that $M \cap S_{N} \neq \emptyset$. In fact, if $a_{N} \in M$, then we are done. If $a_{N} \notin M$, then $I_{R}\left(a_{N}, M\right)=R$. In other words, there is $x \in R$ and $m \in M$ such that $-a_{N} x+m=1$; that is $1+a_{N} x \in M$. By the definition of $S_{N}, 1+a_{N} x \in S_{N}$. Hence $1+a_{N} x \in M \cap S_{N}$. Thus $M \cap S_{N} \neq \emptyset$.

Hence $P_{N} \subset M$, the inclusion being strict, and so we have found a chain of prime ideals of length $N+1$ :

$$
P_{0} \subset P_{1} \subset \cdots \subset P_{N} \subset M \text {. }
$$

We conclude that the Krull dimension of $R$ is at least $N+1$. 
The proof that the Krull dimension of $\mathbb{R}\left[x_{1}, \ldots, x_{n}\right]$ is $n$ now works as in Coquand and Lombardi's paper:

Proposition 3.4 (Coquand-Lombardi). Let $F$ be a field and $R \neq\{0\}$ a commutative unital algebra over $F$. If any $(n+1)$-tupel $\left(f_{0}, \ldots, f_{n}\right) \in R^{n+1}$ is algebraically dependent over $F$, that is, if there is a non-zero polynomial $Q \in F\left[y_{0}, \ldots, y_{n}\right]$ such that $Q\left(f_{0}, \ldots, f_{n}\right)=0$, then the Krull dimension of $R$ is at most $n$.

Proof. Let $Q\left(f_{0}, \ldots, f_{n}\right)=0$ for some non-zero polynomial $Q \in F\left[y_{0}, \ldots, y_{n}\right]$. We assume that the monomials are ordered lexicographically with respect to the powers $\left(i_{0}, i_{1}, \ldots, i_{n}\right) \in$ $\mathbb{N}^{n+1}$. This means that $\left(i_{0}, i_{1}, \ldots, i_{n}\right) \preceq\left(j_{0}, j_{1}, \ldots, j_{n}\right)$ if either $i_{0}<j_{0}$ or if there is $m \in\{0, \ldots, n-1\}$ such that $i_{\nu}=j_{\nu}$ for all $\nu$ with $0 \leq \nu \leq m$ and $i_{m+1}<j_{m+1}$. Let

$$
a_{i_{0}, \ldots, i_{n}} f_{0}^{i_{0}} f_{1}^{i_{1}} \ldots f_{n}^{i_{n}}
$$

be the "first" monomial appearing in the relation above (here the coefficient $a_{i_{0}, \ldots, i_{n}}$ belongs to $F$ and $\left.\left(i_{0}, \ldots, i_{n}\right) \in \mathbb{N}^{n+1}\right)$. Without loss of generality we may assume that the coefficient of this monomial is 1 . Then $Q\left(f_{0}, \ldots, f_{n}\right)$ can be written as

$$
\begin{aligned}
Q=f_{0}^{i_{0}} \ldots f_{n-1}^{i_{n-1}} f_{n}^{i_{n}} & +f_{0}^{i_{0}} \ldots f_{n-1}^{i_{n-1}} f_{n}^{1+i_{n}} R_{n}+f_{0}^{i_{0}} \ldots f_{n-1}^{1+i_{n-1}} R_{n-1}+\ldots \\
& +f_{0}^{i_{0}} f_{1}^{1+i_{1}} R_{1}+f_{0}^{1+i_{0}} R_{0}
\end{aligned}
$$

where $R_{j}$ belongs to $F\left[f_{j}, f_{j+1}, \ldots, f_{n}\right], j=0,1, \ldots, n$. Hence $Q$ has been written in the form given by equation 3.1 (with $a_{j}:=f_{j}$ and $x_{j}:=R_{j}$ ), that is

$$
f_{0}^{i_{0}}\left(f_{1}^{i_{1}}\left(\cdots\left(f_{n}^{i_{n}}\left(1+f_{n} R_{n}\right)+\cdots\right)\right)+f_{0} R_{0}\right)=0 .
$$

We conclude from Theorem 3.3, that the Krull dimension of $R$ is at most $n$.

In order to show that $\mathbb{R}\left[x_{1}, \ldots, x_{n}\right]$ satisfies the assumptions of Proposition 3.4 and to deduce its Krull dimension, we need some additional information.

Proposition 3.5. Let $R=F\left[x_{1}, \ldots, x_{n}\right]$. Then the ideals $I_{R}\left(x_{1}\right), I_{R}\left(x_{1}, x_{2}\right), \ldots, I_{R}\left(x_{1}, \ldots, x_{j}\right)$ with $1 \leq j \leq n$ are prime ideals.

Proof. We may assume that $1 \leq j<n$, since the ideals $I_{R}\left(x_{1}, \ldots, x_{n}\right)$ are maximal, hence prime. First we observe that $F\left[x_{1}, \ldots, x_{n}\right]=F[\boldsymbol{x}]\left[x_{1}, \ldots, x_{j}\right]$, the polynomial ring with indeterminates $x_{1}, \ldots, x_{j}$ and coefficients from the ring $F[\boldsymbol{x}]$, where $\boldsymbol{x}=\left(x_{j+1}, \ldots, x_{n}\right)$. Then every $f \in R$ can uniquely be written as

$$
f\left(x_{1}, \ldots, x_{n}\right)=\sum a_{\ell_{1}, \ldots \ell_{j}} x_{1}^{\ell_{1}} \ldots x_{j}^{\ell_{j}},
$$

where $a_{\ell_{1}, \ldots, \ell_{j}} \in F[\boldsymbol{x}]$.

Let us now consider the surjective ring-homomorphism

$$
h:\left\{\begin{aligned}
F\left[x_{1}, \ldots, x_{n}\right] & \rightarrow F[\boldsymbol{x}] \\
f\left(x_{1}, \ldots, x_{n}\right) & \mapsto \underbrace{a_{0, \ldots, 0}}_{j}\left(x_{j+1}, \ldots, x_{n}\right),
\end{aligned}\right.
$$


where $a_{0, \ldots, 0}\left(x_{j+1}, \ldots, x_{n}\right)$ is the coefficient of the monomial $x_{1}^{0} \ldots x_{j}^{0}$. Then the kernel of $h$ is the union of the zero-polynomial with the set of polynomials in $F\left[x_{1}, \ldots, x_{n}\right]$ all of whose summands contain at least one of the indeterminates $x_{1}, \ldots, x_{j}$.

We conclude that the kernel of $h$ coincides with the ideal $I:=I_{R}\left(x_{1}, \ldots, x_{j}\right)$. Hence $R / I$ is isomorphic to $F[\boldsymbol{x}]$. Since $F[\boldsymbol{x}]$ is an integral domain, we conclude that $I$ is a prime ideal.

Proposition 3.6. Let $F$ be a field. The dimension of the $F$-vector space $V_{m}\left(x_{1}, \ldots, x_{n}\right)$ of all polynomials $p \in F\left[x_{1}, \ldots, x_{n}\right]$ with $\operatorname{deg} p \leq m$ is $\left(\begin{array}{c}n+m \\ n\end{array}\right)$.

Proof. We proceed by induction on $n$. If $n=1$, then

$$
V_{m}(x)=\left\{\sum_{j=0}^{m} a_{j} x^{j}: a_{j} \in F\right\}
$$

has dimension $m+1$, which coincides with $\left(\begin{array}{c}1+m \\ 1\end{array}\right)$. Now suppose that the formula holds for all $\nu$ with $1 \leq \nu \leq n$. If $x_{1}^{j_{1}} \ldots x_{n}^{j_{n}} x_{n+1}^{j_{n+1}}$ is a monomial with $\sum_{i=1}^{n+1} j_{i} \leq m$, then for fixed $j:=j_{n+1} \in\{0,1, \ldots, m\}$ we necessarily must have $\sum_{i=1}^{n} j_{i} \leq m-j$. Hence, by induction hypothesis, we have $\left(\begin{array}{c}n+m-j \\ n\end{array}\right)$ possibilities to choose these exponents $j_{1}, \ldots, j_{n}$. On the whole, we have

$$
L:=\sum_{j=0}^{m}\left(\begin{array}{c}
n+m-j \\
n
\end{array}\right)=\left(\begin{array}{l}
n \\
n
\end{array}\right)+\left(\begin{array}{c}
n+1 \\
n
\end{array}\right)+\cdots+\left(\begin{array}{c}
n+m \\
n
\end{array}\right)
$$

choices. But, $L=\left(\begin{array}{c}n+1+m \\ n+1\end{array}\right)$. Thus we are done.

Remark 3.7. We also obtain that there are exactly $\left(\begin{array}{c}n+m \\ n\end{array}\right)$ tuples $\left(j_{1}, \ldots, j_{n}\right) \in \mathbb{N}^{n}$ with $\sum_{j=1}^{n} j_{i} \leq m$.

The following result is due to Perron [11]. We present a proof given to us by Witold Jarnicki.

Theorem 3.8 (Perron). Let $p_{1}, \ldots, p_{n+1}$ be polynomials in $F\left[x_{1}, \ldots, x_{n}\right]$. Then there exists a non-zero polynomial $P \in F\left[y_{1}, \ldots, y_{n+1}\right]$ in $n+1$ variables such that

$$
P\left(p_{1}, \ldots, p_{n+1}\right)=0 .
$$

Proof. We may assume that the polynomials $p_{j}$ are different from 0 (otherwise take $P\left(y_{1}, \ldots, y_{n+1}\right)=y_{n_{0}}$, where $p_{n_{0}} \equiv 0$.) Let $k:=1+\max _{1 \leq j \leq n+1} \operatorname{deg} p_{j}$. For $\operatorname{big} L \in \mathbb{N}$, to be determined later, we are looking for $P \in F\left[y_{1}, \ldots, y_{n+1}\right]$ with $0 \leq \operatorname{deg} P \leq L$ and $P\left(p_{1}, \ldots, p_{n+1}\right)=0$.

Let $V$ be the vector space of all polynomials $p$ in $F\left[x_{1}, \ldots, x_{n}\right]$ with $\operatorname{deg} p \leq k L$. Then, by Proposition 3.6,

$$
\operatorname{dim} V=\left(\begin{array}{c}
k L+n \\
n
\end{array}\right)=: A(L)
$$

\footnotetext{
${ }^{1}$ For example in the case $j=2$ and $n=3, x_{1} x_{2} \mapsto 0, x_{1} x_{2} x_{3} \mapsto 0$ and $x_{2}+x_{3} \mapsto x_{3}$.
} 
Consider now the following collection $\mathcal{C}$ of polynomials:

$$
p_{1}^{j_{1}} \ldots p_{n+1}^{j_{n+1}}: j_{i} \in \mathbb{N}, \sum_{i=1}^{n+1} j_{i} \leq L .
$$

Note that at this point we do not yet consider the set of these polynomials, because they may not be pairwise distinct.

Each member of $\mathcal{C}$ belongs to $V$, because for $p \in \mathcal{C}$,

$$
\operatorname{deg} p \leq k\left(j_{1}+\cdots+j_{n+1}\right) \leq k L .
$$

If two members of $\mathcal{C}$ coincide, say

$$
p_{1}^{j_{1}} \ldots p_{n+1}^{j_{n+1}}=p_{1}^{j_{1}^{*}} \ldots p_{n+1}^{j_{n+1}^{*}},
$$

where $\left(j_{1}, \ldots, j_{n+1}\right) \neq\left(j_{1}^{*}, \ldots, j_{n+1}^{*}\right)$, then we let

$$
F\left(y_{1}, \ldots, y_{n+1}\right)=y_{1}^{j_{1}} \ldots y_{n+1}^{j_{n+1}}-y_{1}^{j_{1}^{*}} \ldots y_{n+1}^{j_{n+1}^{*}}
$$

and we are done. So let us assume that all the members of $\mathcal{C}$ are distinct. Let $S$ be the set of all these members from $\mathcal{C}$. Then, by Remark 3.7,

$$
\operatorname{card} S=\left(\begin{array}{c}
L+n+1 \\
n+1
\end{array}\right)=: B(L) .
$$

Recall that $S \subseteq V$. We claim that $B(L)>A(L)$ for some $L$ (depending on $n$ ).

In fact, looking upon $B(L)$ and $A(L)$ as polynomials in $L$, we have that $\operatorname{deg} B=n+1$ and $\operatorname{deg} A=n$. Thus, for large $L$, we obtain that $B(L)>A(L)$.

Thus the cardinal of set $S$ is strictly bigger than the dimension of the vector space $V$ it belongs to. Hence $S$ is a linear dependent set in $V$. In other words, there is a nontrivial linear combination of the elements from $S$ that is identically zero. This implies that there is a non-zero polynomial $P \in F\left[y_{1}, \ldots, y_{n+1}\right]$ of degree at most $L$ such that $P\left(p_{1}, \ldots, p_{n+1}\right)=0$.

Theorem 3.9. If $F$ is a field then the Krull dimension of $F\left[x_{1}, \ldots, x_{n}\right]$ is $n$.

Proof. By Perron's Theorem 3.8, $R:=F\left[x_{1}, \ldots, x_{n}\right]$ satisfies the assumption of Proposition 3.4. Hence the Krull dimension of $R$ is less than or equal to $n$. By Proposition 3.5, we have a chain of prime ideals

$$
\{0\} \subset I_{R}\left(x_{1}\right) \subset I_{R}\left(x_{1}, x_{2}\right) \subset \cdots \subset I_{R}\left(x_{1}, \ldots, x_{n}\right) .
$$

Since $\{0\}$ is a prime ideal too, this chain has length $n$. Thus the Krull dimension of $R$ is $n$.

\section{Anderson's approach to Noether's minimal prime theorem}

To prove Bass' Theorem along the lines developed by Estes and Ohm [4], we need to collect in the following classical Theorem by E. Noether some information on the abundance of minimal prime ideals in Noetherian rings. We present a recent proof developed by D. Anderson [1]. 
Theorem 4.1. Let $R$ be a Noetherian ring. Then the system, $\mathscr{P}_{\text {min }}$, of prime ideals containing a given proper ideal $I \subseteq R$ and that are minimal (with respect to set inclusion) is a non-empty finite set.

Proof. Let $\mathscr{P}_{I}$ be the set of all prime ideals containing $I$. Then $\mathscr{P}_{I} \neq \emptyset$, because there exists (using Zorn's Lemma) a maximal ideal $M$ containing $I$. Obviously $M$ is prime. A second use of Zorn's Lemma shows that $\mathscr{P}_{I}$ also admits minimal elements. Hence $\mathscr{P}_{\text {min }} \neq \emptyset .^{2}$

Next we consider the set $\mathfrak{J}$ of all ideals of the form

$$
P_{1} \odot \cdots \odot P_{n}:=\left\{\sum_{j=1}^{m} f_{1, j} \ldots f_{n, j}: f_{k, j} \in P_{k}, m \in \mathbb{N}\right\},
$$

where $P_{k} \in \mathscr{P}_{\text {min }}, n \in \mathbb{N}$. Since $R$ is a Noetherian ring, every ideal in $\mathfrak{J}$ is finitely generated.

Case 1 If for some $J:=P_{1} \odot \cdots \odot P_{n_{0}} \in \mathfrak{J}$ we have $J \subseteq I$, then $P_{1} \cdots P_{n_{0}} \subseteq I \subseteq P$ for every $P \in \mathscr{P}_{\text {min }}$. Hence, the primeness of $P$ implies that there is $i_{0} \in\left\{1, \ldots, n_{0}\right\}$ depending on $P$, such that $P_{i_{0}} \subseteq P$ (for if this is not the case, there exists for every $j \in\left\{1, \ldots, n_{0}\right\}$ an element $f_{j} \in P_{j} \backslash P$ with $f_{1} \cdots f_{n} \in I \subseteq P$, contradicting the primeness of $P$ ). Since $P$ is minimal, $P=P_{i_{0}}$. Hence $\mathscr{P}_{\text {min }}=\left\{P_{1}, \ldots, P_{n_{0}}\right\}$ and we are done.

Case 2 Let us suppose that $J \nsubseteq I$ for every $J \in \mathfrak{J}$. The aim is to show that this case does not occur. Consider the set

$$
\mathscr{L}:=\{L \subseteq R, L \text { ideal, } I \subseteq L, J \nsubseteq L \text { for each } J \in \mathfrak{J}\} .
$$

Then $\mathscr{L} \neq \emptyset$ because, by assumption, $L:=I \in \mathscr{L}$. Moreover all ideals in $\mathscr{L}$ are proper, because $J \subseteq R$ for all $J \in \mathfrak{J}$. With respect to set inclusion, $\mathscr{L}$ is partially ordered. We claim that

$$
\mathscr{L} \text { admits a maximal element } M \text { and } M \text { is automatically prime. }
$$

Suppose for the moment that this has been verified. Then, using Zorn's Lemma, there exists a minimal prime ideal $P$ over $I$ (hence $P \in \mathfrak{J}$ ) with $I \subseteq P \subseteq M$. This is a contradiction, though, to the fact that $M \in \mathscr{L}$. We conclude that this second case cannot occur. Hence, in view of the first case, $\mathscr{P}_{\min }$ is finite.

Let us verify the two assertions in (4.1). To this end, let $\left\{L_{\lambda}: \lambda \in \Lambda\right\}$ be an increasing chain in $\mathscr{L}$. Let us show that $L:=\bigcup_{\lambda} L_{\lambda} \in \mathscr{L}$.

i) $I \subseteq L$ is obviously satisfied and $L$ is an ideal because the chain is increasing.

ii) Let $J \in \mathfrak{J}$. Note that $J$ is finitely generated, say $J=I_{R}\left(f_{1}, \ldots, f_{d}\right)$, and that $J \nsubseteq L_{\lambda}$ for any $\lambda$. If we suppose (in view of achieving a contradiction) that $J \subseteq \bigcup_{\lambda \in \Lambda} L_{\lambda}=L$, then $f_{j} \in L_{\lambda_{j}}$ for some $\lambda_{j} \in \Lambda,(j=1, \ldots, d)$. The monotonicity of the chain implies that

\footnotetext{
${ }^{2}$ For later purposes we note that, by the same reason, if $I$ and $P$ are ideals, $P$ prime and $I \subseteq P$, then there exist minimal prime ideals $P_{\min }$ with $I \subseteq P_{\min } \subseteq P$.
} 
there exists $\lambda^{\prime}$ such that $f_{j} \in L_{\lambda^{\prime}}$ for all $j=1, \ldots, d$. Thus $J \subseteq L_{\lambda^{\prime}}$, a contradiction to the hypothesis that $L_{\lambda^{\prime}} \in \mathscr{L}$.

Thus we have shown that $\mathscr{L}$ is an inductive set and so, by Zorn's Lemma, $\mathscr{L}$ admits a maximal element $M$. In particular, $I \subseteq M$. We claim that $M$ is prime.

To see this, we first observe that $M$ is proper since otherwise $J \subseteq M=R$ for every $J \in \mathfrak{J}$. Now let $f, g \in R$ with $f g \in M$. Suppose, to the contrary, that $f \notin M$ and $g \notin M$. Since $M$ is a maximal element in $\mathscr{L}$, and $I \subseteq M$, there is $J_{f} \in \mathfrak{J}$ and $J_{g} \in \mathfrak{J}$ such that

$$
J_{f} \subseteq I_{R}[f, M] \text { and } J_{g} \subseteq I_{R}(g, M) .
$$

By the definition of $\mathfrak{J}$, there exists $P_{i}$ and $P_{k}$ in $\mathscr{P}_{\min }$ with $I \subseteq P_{i} \subseteq J_{f}$ and $I \subseteq P_{k} \subseteq J_{g}$. We claim that

$$
P_{i} \cdot P_{k} \subseteq I_{R}(f g, M) \subseteq M .
$$

In fact, if $p_{i} \in P_{i}$ and $p_{k} \in P_{k}$, then there are $x_{i}, x_{k} \in R$ and $m_{i}, m_{k} \in M$ such that

$$
p_{i} p_{k}=\left(x_{i} f+m_{i}\right)\left(x_{k} g+m_{k}\right)=x_{i} x_{k}(f g)+m_{i}\left(x_{k} g\right)+m_{k}\left(x_{i} f\right)+m_{i} m_{k} \in M \text {. }
$$

Because $M$ is an ideal, $P_{i} \odot P_{k} \subseteq M$. Thus we have found an element in $\mathfrak{J}$ that is contained in $M$. Since $M \in \mathscr{L}$, this is a contradiction. This proves that $M$ is prime.

\section{The Estes-Ohm Approach}

Here we present the approach to Bass' Theorem given by Estes and Ohm [4].

Lemma 5.1. Let $R$ be a commutative ring and $I_{0}, I_{1}, I_{2}$ three ideals with $I_{0} \subseteq I_{1} \cup I_{2}$. Then $I_{0} \subseteq I_{1}$ or $I_{0} \subseteq I_{2}$.

Proof. Suppose that neither $I_{0} \subseteq I_{1}$ nor $I_{0} \subseteq I_{2}$. Then there are $a_{1} \in I_{0} \backslash I_{2} \subseteq I_{1}$ and $a_{2} \in I_{0} \backslash I_{1} \subseteq I_{2}$. Since $I_{0}$ is an ideal, $s:=a_{1}+a_{2} \in I_{0} \subseteq I_{1} \cup I_{2}$. Without loss of generality we may assume that $s \in I_{1}$. Then $a_{2}=s-a_{1} \in I_{1}$; a contradiction. We conclude that $I_{0} \subseteq I_{1}$ or $I_{0} \subseteq I_{2}$.

Remark 5.2. The assertion above does not hold (in general) for unions of three (or more) ideals. In fact, let $R$ be a finite ring such that not all of its maximal ideals are principal. Let $I \subseteq R$ be a non-principal maximal ideal. Then $N=$ card $I \geq 4$ and $I=\bigcup_{j=1}^{N} R x_{j}$. But of course, $I_{j}:=R x_{j}$ does not contain $I$. A specific example is, for instance, the quotient ring $R=\mathbb{Z}_{2}[x, y] / M$ where $M$ is the ideal generated by $x^{2}, x y$ and $y^{2}$. When denoting the equivalence class of $u \in \mathbb{Z}_{2}[x, y]$ by $\tilde{u}$, we have

$$
R=\{\tilde{0}, \tilde{1}, \tilde{x}, \tilde{y}, \tilde{x}+\tilde{y}, \tilde{1}+\tilde{x}+\tilde{y}\},
$$

and as $I$ we may take $I=I_{R}(\tilde{x}, \tilde{y})$, which coincides with the set $\{\tilde{0}, \tilde{x}, \tilde{y}, \tilde{x}+\tilde{y}\}$.

If one stipulates, however, that the ideals $I_{j}$ are prime, then one obtains the following well-known result. For the readers' convenience we present its proof, too.

Lemma 5.3. Let $R$ be a commutative ring and $I, P_{1}, \ldots, P_{m}$ ideals in $R$ such that each $P_{j}$ is prime and

$$
I \subseteq \bigcup_{j=1}^{m} P_{j} .
$$

Then $I \subseteq P_{j_{0}}$ for some $j_{0} \in\{1, \ldots, m\}$. 
Proof. If $m=1$, then nothing has to be shown. If $m=2$, then the assertion holds by Lemma 5.1. So we may assume that $m \geq 3$. For $j \in\{1, \ldots, m\}$, let

$$
Q_{j}=\bigcup_{k \neq j} P_{k} .
$$

We claim that there is $j$ such that $I \subseteq Q_{j}$. Suppose, to the contrary, that for every $j, I \nsubseteq Q_{j}$. Then we may choose for $j \in\{1, \ldots, m\}$ elements $a_{j} \in I \backslash Q_{j}$. Note that $I \backslash Q_{j} \subseteq P_{j}$. Let

$$
b_{j}=\prod_{k \neq j} a_{k} .
$$

Then $b_{j} \in I$ and $b_{1}+\cdots+b_{m} \in I \subseteq \bigcup_{k=1}^{m} P_{k}$. Hence there is $j \in\{1, \ldots, m\}$ such that $b_{1}+\cdots+b_{m} \in P_{j}$. Since $b_{k} \in P_{j}$ for every $k \neq j$, we deduce that $\sum_{k \neq j} b_{k} \in P_{j}$. Therefore $b_{j} \in P_{j}$. Since $P_{j}$ is prime, there is $k \neq j$ such that $a_{k} \in P_{j} \subseteq Q_{k}$. This is a contradiction to the choice of the elements $a_{1}, \ldots, a_{m}$. We conclude that $I \subseteq \bigcup_{k \neq j} P_{k}$ for some $j$.

Now we proceed by backwards induction to reduce the number of prime ideals up to the case $m=2$. That case, though, is handled by Lemma 5.1 .

Lemma 5.4. Let $P_{j}$ be prime ideals in a commutative unital ring $R$ with $P_{j} \nsubseteq P_{k}$ for $j \neq k, j, k \in\{1, \ldots, m\}$. Let $a, r \in R$. Then there exists $b \in R$ such that for all $j \in\{1, \ldots, m\}$ :

$$
\text { if } a \notin P_{j} \text { then } r+a b \notin P_{j} \text {. }
$$

Proof. If $r \in \bigcap_{j=1}^{m} P_{j}$, then we may choose for $b$ any element not in $\bigcup_{j=1}^{m} P_{j}$. If $r \notin$ $\bigcup_{j=1}^{m} P_{j}$, then we take $b_{j} \in P_{j}$ and let $b=\prod_{j=1}^{m} b_{j}$. In the remaining cases, modulo a re-enumeration, we may assume that $r \in \bigcap_{j=1}^{s} P_{j}$ but $r \notin \bigcup_{j=s+1}^{m} P_{j}$. By Lemma 5.3, the hypothesis $P_{j} \nsubseteq P_{k}$ for $j \neq k$ implies that $P_{j} \nsubseteq \subseteq \bigcup_{k=1}^{s} P_{k}$ for each $j \in\{s+1, \ldots, m\}$. Let $b_{j} \in P_{j} \backslash \bigcup_{k=1}^{s} P_{k}, j \geq s+1$, and let

$$
b=b_{s+1} \cdot \cdots \cdot b_{m} .
$$

Then $b \in \bigcap_{j=s+1}^{m} P_{j}$. But $b \notin P_{k}$ for $1 \leq k \leq s$, because otherwise the primeness of $P_{k}$ implies that one of the factors $b_{j}$ with $s+1 \leq j \leq m$ belongs to $P_{k}$, a contradiction to the choice of $b_{j}$.

Fix $j_{0} \in\{1, \ldots, m\}$ and assume that $a \notin P_{j_{0}}$. We claim that $r+a b \notin P_{j_{0}}$. In fact, assuming the contrary, let $u:=r+a b \in P_{j_{0}}$. If $1 \leq j_{0} \leq s$, then $r \in P_{j_{0}}$, hence $a b=u-r \in P_{j_{0}}$. The assumption on $a$ and the primeness of $P_{j_{0}}$ imply that $b \in P_{j_{0}}$, a contradiction. If $s+1 \leq j_{0} \leq m$, then $b \in P_{j_{0}}$. Hence $r=u-a b \in P_{j_{0}}$; this is a contradiction to the choice of $r$.

Lemma 5.5. Let $R$ be a Noetherian ring. Given $a, a_{j} \in R, j=0,1, \ldots, s$, there exists $b_{j} \in R, j=1, \ldots, s$, such that for $i=1,2, \ldots, s$, the (finite) set, $\mathscr{P}_{i-1}$, of minimal prime ideals $P$ containing

$$
I_{R}\left(a_{0}, a_{1}+b_{1} a, \ldots, a_{i-1}+b_{i-1} a\right)^{3}
$$

\footnotetext{
${ }^{3}$ If $i=1$, then we consider $I_{R}\left(a_{0}\right)$.
} 
has the following property:

$$
\text { if } a \notin P \text {, where } P \in \mathscr{P}_{i-1} \text {, then } a_{i}+b_{i} a \notin P \text {. }
$$

Proof. To prove the assertions via induction, we will use several times Lemma 5.4.

$\underline{i=1}$ : Recall that $\mathscr{P}_{0}$ is the class of all minimal prime ideals $P$ with $I_{R}\left(a_{0}\right) \subseteq P$. By Theorem 4.1, $\mathscr{P}_{0}$ is finite; say $\mathscr{P}_{0}=\left\{P_{1}, \ldots, P_{t}\right\}$. To apply Lemma 5.4, we let $r=a_{1}$. This gives $b_{1} \in R$ such that for all $\nu \in\{1, \ldots, t\}$

$$
a_{1}^{\prime}:=a_{1}+b_{1} a \notin P_{\nu} \text { whenever } a \notin P_{\nu} .
$$

Thus (5.2) is satisfied.

Now let us suppose that $b_{1}, \ldots, b_{i}$ have been constructed and that (5.2) is satisfied for some $i \in\{1, \ldots, s-1\}$. Let $a_{j}^{\prime}:=a_{j}+b_{j} a$, where $j \in\{1, \ldots, i\}$.

$\underline{i \rightarrow i+1}$ : By definition, $\mathscr{P}_{i}$ is the class of all minimal prime ideals $P$ with

$$
I_{R}\left(a_{0}, a_{1}^{\prime}, \ldots, a_{i}^{\prime}\right) \subseteq P .
$$

By Theorem 4.1, $\mathscr{P}_{i}$ is finite. To apply Lemma 5.4, we let $r=a_{i+1}$. This gives $b_{i+1} \in R$ such that for all $P \in \mathscr{P}_{i}$ :

$$
a_{i+1}^{\prime}:=a_{i+1}+b_{i+1} a \notin P \text { whenever } a \notin P .
$$

Thus (5.2) is satisfied for $i+1$.

Theorem 5.6 (Bass). Let $R$ be a Noetherian ring with Krull dimension less than or equal to $n$. Then bsr $R \leq n+1$.

Proof. This is the proof given by Estes and Ohm [4]. Let $\boldsymbol{a}:=\left(a_{1}, \ldots, a_{n+1}, a\right) \in U_{n+2}(R)$. We have to show that $\boldsymbol{a}$ is reducible. Choose $a_{0}=0$. Associate with $a_{j}$ the elements $b_{j}$ comming from Lemma $5.5, j=1, \ldots, n+1$, and let $a_{j}^{\prime}=a_{j}+b_{j} a$. For $i=1, \ldots, n+1$, consider the ideals

$$
I_{i}:=I_{R}\left(a_{0}, a_{1}^{\prime}, \ldots, a_{i}^{\prime}\right),
$$

and let $\mathscr{P}_{i}$ be the (finite) set of minimal prime ideals $P$ with $I_{i} \subseteq P$.

Note that the reducibility of $\boldsymbol{a}$ is a consequence to the assertion that $I_{n+1}=R$. Suppose, to the contrary, that $I_{n+1}$ is a proper ideal.

We claim that

$$
a \notin P \text { for every } P \in \mathscr{P}_{n+1}
$$

In fact, if we suppose that $a \in P$ for some $P \in \mathscr{P}_{n+1}$, then the invertibility of $\boldsymbol{a}$ implies that

$$
P \supseteq I_{R}\left(a_{1}^{\prime}, \ldots, a_{n+1}^{\prime}, a\right)=I_{R}\left(a_{1}, \ldots, a_{n+1}, a\right)=R,
$$

a contradiction to the fact that prime ideals are proper ideals. Hence assertion 5.3 holds.

Consider now the following chain of ideals:

$$
I_{R}\left(a_{0}\right) \subseteq I_{R}\left(a_{0}, a_{1}^{\prime}\right) \subseteq \ldots \subseteq I_{R}\left(a_{0}, a_{1}^{\prime}, \ldots, a_{n}^{\prime}\right) \subseteq I_{R}\left(a_{0}, a_{1}^{\prime}, \ldots, a_{n+1}^{\prime}\right)=I_{n+1} \subset R .
$$

We shall construct a chain of prime ideals that has length $n+1$ (in other words $n+2$ elements) which will yield a contradiction to the assumption that the Krull dimension of $R$ is less than or equal to $n$.

Let $P_{n+1} \in \mathscr{P}_{n+1}$. Choose a minimal prime ideal $P_{n} \in \mathscr{P}_{n}$ with

$$
I_{R}\left(a_{0}, a_{1}^{\prime}, \ldots, a_{n}^{\prime}\right) \subseteq P_{n} \subseteq P_{n+1} .
$$


Backwards induction yields minimal prime ideals $P_{i}$ with

$$
I_{R}\left(a_{0}, a_{1}^{\prime}, \ldots, a_{i}^{\prime}\right) \subseteq P_{i} \subseteq P_{i+1} \subseteq \ldots \subseteq P_{n+1}
$$

$(i=1, \ldots, n)$, and finally a minimal prime ideal $P_{0}$ with

$$
I_{R}\left(a_{0}\right) \subseteq P_{0} \subseteq P_{1}
$$

We claim that all the inclusions in the chain

$$
P_{0} \subset P_{1} \subset \cdots \subset P_{n} \subset P_{n+1}
$$

are strict. To do so, we use Lemma 5.5. Fix $i \in\{1, \ldots, n+1\}$ and consider $I_{i-1}=I_{R}\left(a_{0}, a_{1}^{\prime}, \ldots, a_{i-1}^{\prime}\right)$, with the convention that $I_{0}=I_{R}\left(a_{0}\right)$. We first observe that $a \notin P_{i-1}$, since otherwise $a \in P_{n+1}$, a contradiction to (5.3).

Hence, by Lemma 5.5, $a_{i}^{\prime} \notin P_{i-1}$. But by construction, $a_{i}^{\prime} \in P_{i}$. Thus $P_{i-1} \subset P_{i}$, the inclusion being strict. Hence, under the assumption that $I_{n+1}$ is proper, we have shown that the Krull dimension of $R$ is at least $n+1$. This contradicts the hypothesis. Consequently, $I_{n+1}=R$. Thus, as already mentioned, $\boldsymbol{a} \in U_{n+2}(R)$ is reducible. Hence $\operatorname{bsr} R \leq n+1$.

To conclude this section, let us mention the following generalization of Bass' Theorem given by R. Heitmann [7]. It shows that the Noetherian condition can be dropped.

Theorem 5.7. Let $R$ be a commutative unital ring with Krull dimension $n$. If $R$ is an integral domain, then bsr $R \leq n+1$. If, on the other hand, $R$ has zero-divisors, then bsr $R \leq n+2$.

\section{The topological Stable RANK of $\mathbb{R}\left[x_{1}, \ldots, x_{n}\right]$}

Definition 6.1. Let $R$ be a ring endowed with a topology $\mathcal{T}$ (we do not assume that the topology is compatible with the algebraic operations + and $\cdot)$. The topological stable rank, $\operatorname{tsr}_{\mathcal{T}} R$, of $(R, \mathcal{T})$ is the least integer $n$ for which $U_{n}(R)$ is dense in $R^{n}$, or infinite if no such $n$ exists.

If the ring $R$ is endowed with two topologies $\mathcal{T}_{1}$ and $\mathcal{T}_{2}$ such that $\mathcal{T}_{1}$ is weaker than $\mathcal{T}_{2}$, then

$$
\operatorname{tsr}_{\mathcal{T}_{1}} R \leq \operatorname{tsr}_{\mathcal{T}_{2}} R
$$

For the ring of polynomials, we work with the topology of uniform convergence.

Theorem 6.2. The topological stable rank of $\mathbb{R}\left[x_{1}, \ldots, x_{n}\right]$ is $n+1$.

Proof. We first prove that

$$
\operatorname{tsr} \mathbb{R}\left[x_{1}, \ldots, x_{n}\right] \leq n+1 .
$$

Let $\boldsymbol{p}:=\left(p_{1}, \ldots, p_{n+1}\right)$ be an $(n+1)$-tuple in $\mathbb{R}\left[x_{1}, \ldots, x_{n}\right]$. Now we look upon $\boldsymbol{p}$ as being an $(n+1)$-tuple in $\mathbb{C}\left[z_{1}, \ldots, z_{n}\right]$. Choose, according to Perron's Theorem 3.8, a non-zero polynomial $P$ over $\mathbb{C}$ with $n+1$ indeterminates such that

$$
P\left(p_{1}, \ldots, p_{n+1}\right)=0 .
$$


Then $P$, looked upon as a polynomial function, vanishes identically on the image $\boldsymbol{p}\left(\mathbb{C}^{n}\right)$. But $P$ cannot vanish identically on the ball

$$
B(\mathbf{0}, \varepsilon):=\left\{\boldsymbol{x}=\left(x_{1}, \ldots, x_{n+1}\right) \in \mathbb{R}^{n+1}:\|\boldsymbol{x}\|_{2} \leq \varepsilon\right\},
$$

since otherwise $P$ would be the zero-polynomial (just consider the partial derivatives at the origin). Since $\varepsilon>0$ can be chosen arbitrarily small, we obtain a null-sequence $\left(\varepsilon_{k}\right)$ in $\mathbb{R}^{n+1}$ such that $\varepsilon_{k} \notin \boldsymbol{p}\left(\mathbb{C}^{n}\right)$. Hence the $(n+1)$-tuple $\boldsymbol{p}-\varepsilon_{k}$ is invertible in $C\left(\mathbb{C}^{n}, \mathbb{C}\right)$. From Theorem 1.3 we deduce that $\boldsymbol{p}-\boldsymbol{\varepsilon}_{k}$ is in $U_{n+1}\left(\mathbb{R}\left[x_{1}, \ldots, x_{n}\right]\right)$. Since $\boldsymbol{p}-\boldsymbol{\varepsilon}_{k}$ uniformly approximates $\boldsymbol{p}$, we are able to conclude that $\operatorname{tsr} \mathbb{R}\left[x_{1}, \ldots, x_{n}\right] \leq n+1$.

Next we show that tsr $\mathbb{R}\left[x_{1}, \ldots, x_{n}\right] \geq n+1$.

Consider the identity map $\boldsymbol{x}=\left(x_{1}, \ldots, x_{n}\right)$ of $\mathbb{R}^{n}$ onto $\mathbb{R}^{n}$. Note that

$$
\boldsymbol{x} \in \underbrace{\mathbb{R}\left[x_{1}, \ldots, x_{n}\right] \times \cdots \times \mathbb{R}\left[x_{1}, \ldots, x_{n}\right]}_{n \text {-times }} .
$$

Suppose that there exist invertible $n$-tuples in $\mathbb{R}\left[x_{1}, \ldots, x_{n}\right]$ that uniformly approximate $\boldsymbol{x}$. That is, for every $\varepsilon>0$ there is $\boldsymbol{f}=\left(f_{1}, \ldots, f_{n}\right) \in U_{n}\left(\mathbb{R}\left[x_{1}, \ldots, x_{n}\right]\right)$ such that $\left|x_{j}-f_{j}\right|<\varepsilon$ for $j=1, \ldots, n$. This implies of course that $x_{j}-f_{j}$ is a constant (we keep this generality though, since it also works for non-polynomial rings). In particular, this inequality then holds on the unit sphere $S_{n-1}$. Let $A$ be the Banach algebra $A=$ $C\left(S_{n-1}, \mathbb{R}\right)$ of all continuous, real-valued functions on $S_{n-1}$, endowed with the supremum norm. We obviously have that $\boldsymbol{f} \in U_{n}(A)$.

By a classical theorem in the theory of Banach algebras ([10] or [13]) there exists a matrix $H \in M_{n}(A)$ with

$$
\left(x_{1}, \ldots, x_{n}\right)^{t}=(\exp H)\left(f_{1}, \ldots, f_{n}\right)^{t}
$$

whenever $\varepsilon$ is chosen small enough. Extending the entries of $H$ with the help of Tietze's Theorem to continuous functions on $\mathbb{R}^{n}$ we obtain a zero-free extension of $\left.\boldsymbol{x}\right|_{\partial \mathbf{B}_{n}}$ to $\mathbf{B}_{n}$. As above (see the proof of Theorem 2.3), this contradicts Brouwer's fixed point theorem.

\section{Acknowledgements}

We thank Leonhard Frerick for providing us the reference [12]; Witold Jarnicki for the proof of Theorem 3.8; Amol Sasane for the reference [1] as well as Peter Pflug and Thomas Schick for valuable comments yielding to a proof of $\operatorname{tsr} \mathbb{R}\left[x_{1}, \ldots, x_{n}\right] \leq n+1$ in Theorem 6.2 .

\section{REFERENCES}

[1] D. Anderson, A note on minimal prime ideals, Proc. Amer. Math. Soc. 122 (1994), 13-14. 13, 19

[2] H. Bass, K-theory and stable algebra, Publications Mathématiques de L'I.H.É.S., 22 (1964), 5-60. 1

[3] T. Coquand, H. Lombardi, A short proof for the Krull dimension of a polynomial ring, Amer. Math. Monthly 112 (2005), 826-829. 1, 7

[4] D. Estes, J. Ohm, Stable range in commutative rings, J. of Algebra 7 (1967), 343-362. 1, 7, $13,15,17$

[5] M.R. Gabel, Lower bounds on the stable range of polynomial rings, Pacific J. Math. 61 (1975), 117-120. 8

[6] M. R. Gabel, A.V. Geramita, Stable range for matrices, J. Pure Appl. Algebra 5 (1974), 97-112. 8

[7] R. Heitmann, Generating ideals in Prüfer domains, Pacific J. Math. 62 (1976), 117-126. 18 
[8] I. Kaplansky, Commutative rings, Allyn and Bacon, Boston 1970. 2, 6

[9] S. Lang, Algebra Springer, New York, 2002. 2

[10] T.W. Palmer, Banach algebras and the general theory of *-algebras, Vol 1, Cambridge Univ. Press, London, 1994. 19

[11] O. Perron, Algebra I. Die Grundlagen, 2. Auflage, W. de Gruyter u. Co, Berlin, 1932. 12

[12] A. Płoski, Algebraic dependence of polynomials after O. Perron and some applications, in: Computational commutative and non-commutative algebraic geometry, Proceedings of the NATO Advanced Research Workshop, Chisinau, Republic of Moldova, June 6-11, 2004. Ed.: Cojocaru, Svetlana et al., 167-174 (2005) 19

[13] R. Rupp. Zerofree extension of continuous functions on a compact Hausdorff space, Topology Appl. 93 (1999), 65-71. 19

[14] L. Vasershtein, Stable rank of rings and dimensionality of topological spaces, Funct. Anal. Appl. 5 (1971), 102-110; translation from Funkts. Anal. Prilozh. 5 (1971), No.2, 17-27. 1, 7

Université de Lorraine, Département de Mathématiques et Institut Élie Cartan De Lorraine, UMR 7502, Ile Du Saulcy, F-57045 Metz, France

E-mail address: raymond.mortini@univ-lorraine.fr

Fakultät Allgemeinwissenschaften, Georg-Simon-Ohm-Hochschule Nürnberg, KesslerPlatz 12, D-90489 NürnBerG, GERMAnY

E-mail address: Rudolf.Rupp@ohm-hochschule.de 